\title{
A Usability and Safety Study of Bone-Conduction Headphones During Driving while Listening to Audiobooks
}

\author{
Jasmine Granados, ${ }^{* 1}$ Matthew Hopper ${ }^{2}$ \\ Faculty: Jibo $\mathrm{He}^{1}$ \\ ${ }^{1}$ Department of Psychology, Fairmount College of Liberal Arts and Sciences \\ ${ }^{2}$ Department of Communication Sciences and Disorders, College of Health Professions
}

Bone-conduction technology has been around since the 1800s but using this technology in headphones is relatively new. Bone-conduction transmits sound through the bones of the skull. This research compared the effect of different auditory input methods (bone-conduction versus traditional air-conduction) on driving performance, story comprehension, and subjective workload. Results showed that auditory input method did not significantly affect driving performance or story comprehension. This supports one of the hypotheses that bone-conduction headphones are no more distracting than air-conduction speakers. There were significant differences in workload between driving conditions compared to non-driving conditions but, there was no difference in workload between bone conduction headphones in driving conditions. Bone-conduction headphones do not impair story comprehension and may leave the ear canal open making it a viable option for use while driving. 